\title{
Prácticas y recursos de ocio en la adolescencia
}

\section{Leisure time and resources in adolescents}

\author{
María-Carmen RicoY ${ }^{1}$ \\ cricoy@uvigo.es \\ Jennifer Fernández-Rodríguez \\ jennifer@uvigo.es \\ Universidad de Vigo, España
}

\section{Resumen:}

En general los adolescentes cuentan con muchos recursos y grandes facilidades para disfrutar del ocio, con todo su desarrollo no está exento de problemáticas. Por ello, el objetivo central de este estudio es analizar las actividades y recursos de ocio de los adolescentes, así como determinar los lugares y círculos a los que lo circunscriben. El trabajo presentado se aborda desde un enfoque cualitativo. Para la recogida de información se utilizó la técnica del relato participando 95 adolescentes, de entre 13 y 17 años, que residen en el entorno urbano. Los resultados y conclusiones de la investigación ponen de manifiesto que desarrollan distintas actividades saludables en su tiempo libre a través de recursos variados. Cabe destacar la realización de prácticas de ocio deportivo, las asociadas con la música, así como las de tipo digital. Estas últimas se centran en ver la TV, chatear, navegar por la red y jugar con videojuegos; mientras el uso de las redes sociales adquiere una incidencia moderada. Por otra parte, es de resaltar que muestran preferencia por las actividades llevadas a cabo en solitario y con el grupo de iguales, siendo reducidas

\begin{abstract}
:
In general adolescents have many resources and great facilities to enjoy leisure, but its development is not without problems. Therefore, the aim of this study is to analyze the activities and adolescents' leisure resources, as well as to determine the locations and the circles from which leisure activities arise. This study is tackled from a qualitative approach through a case study. In order to collect data, the reporting technique was used. 95 adolescents aged 13-17 living in urban settings participated in the study. The results and conclusions of the investigation highlight that adolescents develop different healthy activities in their free time through various resources. Activities associated to sports, music and the digital world were especially highlighted as relevant. Among the latter activities, the subject sample mention watching TV, chatting, net surfing and playing video games. Using social networks is not rated as an especially relevant activity. On the other hand, they show preference for activities carried out individually and with other people within their age group. Activities carried out with family members and indoors are not frequently reported.
\end{abstract}

\section{Dirección para correspondencia (correspondence address):}

María-Carmen Ricoy. Dpto. de Didáctica y Organización Escolar. Facultad de Ciencias de la Educación. Universidad de Vigo. Campus Universitario As Lagoas, s/n. 32004 Ourense (España). 
las desarrolladas con la familia y en lugares cerrados. Los hábitos de ocio consumista no se revelan como abusivos.

\section{Palabras clave:}

Adolescentes; prácticas de ocio; tiempo libre; educación.
Consumerist habits do not have a relevant role.

\section{Key words:}

Adolescents; leisure time; free time; education.

\section{Résumé:}

Les adolescents ont généralement beaucoup de ressources et d'excellentes installations pour les loisirs, mais leur développement a parfois des problèmes. Par conséquent, l'objectif principal de cette étude est dianalyser les activités et les ressources pour les loisirs des adolescents et déterminer les lieux et environnments où ils participent. Le travail présenté est abordé sous une approche qualitative. On utilise une technique narrative pour recueillir des informations impliquant 95 adolescents, de 13 à 17 ans vivant en milieu urbain. Les résultats et les conclusions de l'enquête montrent qu'ils développent diverses activités saines dans leur temps libre par le biais de divers moyens. On voit la réalisation des pratiques de loisirs sportives, celles qui sont associées avec de la musique et d'autres de type numérique. On voit bien l'accent mis sur la télé, bavarder, surfer sur le net et jouer à des jeux vidéo; tandis que l'utilisation des réseaux sociaux acquiert une incidence modérée. En outre, il est à noter qu'ils montrent une préférence pour les activités effectuées en solitaire et avec le groupe de pairs, étant assez réduites celles qui se déroulent avec la famille et chez eux. Les habitudes de loisirs consuméristes ne sont pas révélées comme abusives.

\section{Mots clés:}

Adolescents; loisirs; temps libre; éducation.

Fecha de recepción: 8-3-2016

Fecha de aceptación: 23-5-2016

\section{Introducción}

En la actualidad las actividades y los recursos de ocio en la adolescencia han variado mucho con respecto a las generaciones precedentes. La sociedad moderna ofrece una gran diversidad de posibilidades para desarrollar las prácticas de ocio y cada persona puede disfrutar de distinta forma sus experiencias. De un modo paulatino estas actividades se han ido incluyendo como elemento clave de la cotidianidad. De hecho, el ocio constituye un medio esencial en el desarrollo personal, social, cultural y educativo configurándose además como un componente fundamental de calidad de vida.

Entre los elementos que conforman el concepto de ocio se encuentra el de descanso y relajación, primando la ausencia de obligaciones frente a su ingrediente activo donde la actividad se impone (San Martín, 
López \& Esteve, 1999). Con todo, su significado continúa en constante evolución y se halla influenciado, entre otros aspectos, por la revolución tecnológica que promueve el uso globalizado de diferentes recursos y prácticas. Sin embargo, Menchén (2008) sostiene que se generan comportamientos diferenciados sobre el ocio debido a que cada sociedad, comunidad o grupo de personas sigue unas pautas de conducta en su praxis atendiendo a las costumbres y tradiciones. Ello, supone también valorar y respetar la diversidad de manifestaciones y la identidad de los pueblos o colectividades. Además, la existencia de la brecha digital en el acceso y consumo de las tecnologías, desde la familia de origen, es un hecho (Ballesta y Cerezo, 2011). Con todo, en pleno siglo XXI, confluyen manifestaciones análogas a nivel mundial promovidas por los medios digitales como consecuencia de la fuerte influencia y posibilidades de internet.

Por su parte, Noriega et al. (2015) ha reparado en la diferenciación entre el ocio activo (donde el nivel de implicación física y/o mental es elevado) y pasivo (resaltando su vertiente consumista, alienante y sedentaria). Partiendo de las muchas actividades y recursos que posibilita el desarrollo del ocio a las personas, en este trabajo se indaga sobre las que conciernen a los adolescentes. En esta etapa se reconocen diferentes prácticas, entre ellas, cabe mencionar las de tipo educativo-extracurriculares, las promovidas por los medios de comunicación, las asociadas con el descanso y cierto tipo de alimentación ( Gabelas, Marta-Lazo, González, 2015). Otra tipología de experiencias que le pueden resultar gratificantes, al colectivo objeto de estudio, son las que conectan el deporte con la tecnología. Esta alternativa, aparentemente, representa una tendencia en alza frente a las de carácter físico (Mannell, Kaczynski y Aronson, 2005).

Un estudio realizado acerca del bienestar que experimentan los/as adolescentes (Glorieux, Stevens y Vandeweyer, 2005), desvela que para que alcancen un efectivo grado de gratificación es necesario que incorporen el ocio social a su cotidianidad. Además, se argumenta que el nivel de satisfacción interior es más elevado entre las mujeres que practican un ocio más hogareño, que en los varones al centrarse éstos en actividades de interacción social extra-familiar. Cabe resaltar la necesidad de incorporar medidas preventivas, ya que siguiendo a Eimea, Caseya, Harveya, Sawyera, Symonsb y Payneb (2015) la participación en la actividad física disminuye en la adolescencia, particularmente en las chicas.

En la sociedad moderna las prácticas de ocio están presentes en el 
entorno familiar, social y escolar al ser un medio de/para la diversión, relación, relajación y formación. De modo que funcionan como un complemento educativo y de prevención. En general a estas actividades se les atribuyen efectos positivos aunque tampoco hay que obviar las conductas nocivas que pueden llegar a producir, principalmente en colectivos vulnerables. La pubertad es un periodo en el que se desarrollan valores esenciales, rasgos de personalidad y creencias cuya repercusión acompaña, de alguna manera, durante el resto de la existencia (Liu y Hsu, 2013). Por lo tanto, es importante guiar a los/as adolescentes también a través de la realización de las prácticas de ocio para facilitarle el asentamiento de conductas saludables que, además, repercutan positivamente en su rendimiento académico presente y futuro. Un planteamiento multidisciplinar, desde la educación, en el abordaje de tópicos vertebradores, como es el caso, es esencial para fortalecer el desarrollo de conductas positivas, así como para la generación de saberes y prácticas integradoras (Ferreira, Andrade, Fontes, Araújo y Anjos, 2015).

A los/as adolescentes las prácticas de ocio les pueden aportar bienestar y son un vehículo idóneo para el desarrollo normalizado de su personalidad (Douglas et al., 2005). Por ello, procede promover en este colectivo actividades de entretenimiento diversas y valiosas como medio para mejorar su calidad de vida, bagaje sociocultural y educativo. De este modo podrá optimizarse su formación integral, principalmente a través de la educación informal. En un sentido análogo, Castrechini, Pestana y Codina (2015) estiman que el ocio debe ser considerado como uno de los elementos para configurar la identidad personal y sociocultural.

Por otra parte, sobre las tendencias y hábitos de ocio en la infancia y adolescencia, cabe indicar que se supeditan principalmente a espacios abiertos y públicos, privados de uso público y cerrados (Sanz y Romero, 2009). Asimismo Ortega, Lazcano y Rocha (2015) colocan el énfasis en la importancia de la participación en espacios de ocio que promuevan la autonomía y oportunidades de autogestión. Para ello, es necesario incentivar la oferta de las instituciones públicas y de las organizaciones culturales.

Este estudio tiene como finalidad central identificar, analizar y conocer las prácticas de ocio de los/as adolescentes atendiendo a los recursos que utilizan, así como dilucidar las personas con quienes las comparten, los lugares de su desarrollo y su repercusión socioeducativa. Como 
objetivos específicos, bajo un enfoque analítico, se consideraron los siguientes:

- Determinar y estudiar la tipología de actividades de ocio del colectivo.

- Revelar los recursos que usan en el tiempo libre.

- Concretar las personas y/o grupos con quienes comparten el ocio.

- Delimitar los espacios que utilizan para desarrollar las experiencias de ocio.

\section{Método}

Este estudio forma parte de una investigación más amplia. Por su extensión, en este trabajo, se presenta la vertiente cualitativa que se encuadra en la investigación narrativa. Este tipo de estudios puede comprender a una sola persona o a un grupo, en el presente su desarrollo se centra en el análisis de la temática objeto de investigación a partir del alumnado de dos aulas, como subcasos, conformando éstos una realidad singular que presenta múltiples conexiones con otros contextos (Stake, 2010).

\subsection{Participantes}

Para la elección de la muestra se han realizado distintos contactos antes de determinarla. Inicialmente se dialogó con el profesorado de varios Institutos de Educación Secundaria, con el objeto de estudiar las posibilidades de acceso. Finalmente, la selección de la muestra se efectuó mediante un procedimiento de tipo estratégico, atendiendo principalmente a criterios de accesibilidad y considerando la predisposición mostrada por los/as colaboradores/as (Mcmillan y Schumacher, 2005). El muestreo intencional es una peculiaridad de la metodología cualitativa al no tener por objeto la representación estadística.

El total de adolescentes implicados en el estudio fue de 95. Todos ellos cursaban $3^{\circ}$ y $4^{\circ}$ nivel de la Educación Secundaria Obligatoria (ESO) en un Instituto ubicado en una zona urbana de Galicia. Su edad estaba comprendida entre los 13 y 17 años, de los que 37 son varones y 58 mujeres. Del $3^{\circ}$ curso de la ESO han participado 53 alumnos/as (34 mujeres y 19 varones) y de $4^{\circ}$ curso se implicaron 42 estudiantes (24 mujeres y 18 varones). 


\subsection{Instrumento y recogida de información}

El protocolo del relato se ha diseñado ad hoc con la finalidad de dar respuesta a los objetivos planteados en la investigación. Se han obviado, intencionadamente, pautas demasiado restrictivas en el enunciado de la cuestión planteada en el relato para facilitar que aflorase en los/as participantes el discurso, desde la máxima libertad posible. Con esta técnica, se recogió información sobre sus experiencias de ocio, contextualizadas mediante un proceso introspectivo que le otorga pleno significado a las narraciones originadas (Ricoeur, 1995).

Para construir y validar el instrumento se recurrió al juicio de expertos, sometiéndose inicialmente el protocolo del relato a la crítica constructiva de tres especialistas. La selección de éstos se consideró a partir de su experiencia profesional o/y conocimientos respecto al tópico objeto de estudio, así como la proximidad que presentan con el colectivo. Uno de los expertos es profesor de Educación Secundaria, mientras otro es doctor y una doctora en Ciencias de la Educación. A partir de los juicios analíticos y valorativos que ofrecieron se reestructuró el protocolo preliminar. El enunciado de la pregunta del relato quedó finalmente delimitado del siguiente modo: "Describe las actividades que realizas para tu ocio y/o diversión".

La producción de las narrativas fue promovida, por una investigadora, en una reunión con los/as adolescentes en la que se le explicó el interés que presenta la investigación y se les motivó a desarrollar los relatos. Seguidamente se distribuyó el protocolo policopiado entre los/as participantes proporcionándole las consignas necesarias. Para la realización de los relatos se acordó con los/as implicados/as un tiempo máximo de cincuenta minutos. Los respectivos textos los han elaborado en formato papel y en modalidad manuscrita. A partir de esta técnica se recopilaron sus narraciones personales, en las que plasmaron las vivencias y experiencias de forma reflexiva e introspectiva. Su argumentación facilitó la conexión entre distintos aspectos dejando constancia de los hechos y opiniones sobre las prácticas desarrolladas y los recursos de ocio que utilizan. Como datos de identificación se solicitaron los referidos a la edad, sexo y curso académico en el que se encontraban. 


\subsection{Análisis de datos}

A las narrativas obtenidas se le aplicó un análisis de contenido. La categoría principal o de primer nivel de análisis se delimitó a partir de la temática en estudio y de la idea dominante de los relatos. De este modo la noción predominante conformó la categoría principal. Con el software del programa Analysis of Qualitative Data (AQUAD) se desarrolló el análisis de contenido. Para ello, mediante el proceso de codificación de la información se configuró la categorización de primer nivel con sus respectivos subniveles. Este mecanismo permitió reducir los datos brutos en subcategorías representativas, precisar su margen de concentración y dar respuesta a los objetivos del estudio. De este modo, la identificación de los diferentes niveles de categorización se generó a partir de la clasificación de los elementos constitutivos de los respectivos focos, agrupando las ideas análogas que presentan los relatos.

En cuanto a la fiabilidad de los resultados, se ha custodiado el proceso de recogida de información para garantizar la mayor veracidad posible. Por otra parte, se refuerza su consistencia interna mediante los mecanismos técnicos que ofrece el programa informático utilizado. Al facilitar éste una gran sistematización y asegurar una óptima precisión con el análisis. Se siguieron de modo congruente y riguroso los procedimientos para el análisis de contenido (Hernández, Fernández y Baptista, 2006). De este modo, siguiendo a Flores (2003) se proporcionan resultados sólidos de forma estructurada, comprensiva y prolija. Asimismo, se aportan además algunas evidencias textuales, extraídas de las narrativas, en el apartado de resultados.

\section{Resultados}

Estos resultados de índole cualitativa procedentes de las narrativas analizadas, se presentan inicialmente de forma sintética para ofrecer un panorama global. Para ello se han aglutinado, por afinidad, en torno a cuatro dimensiones centrales (figura 1):

- Artística y cultural.

- Tecnológica y/o con juegos.

- Turística y/o deportiva.

- Sedentaria, prácticas sociales y/o con consumo de sustancias. 
A través de los distintos subapartados se exponen sistemática y minuciosamente los resultados cualitativos obtenidos, atendiendo a los extremos referidos.

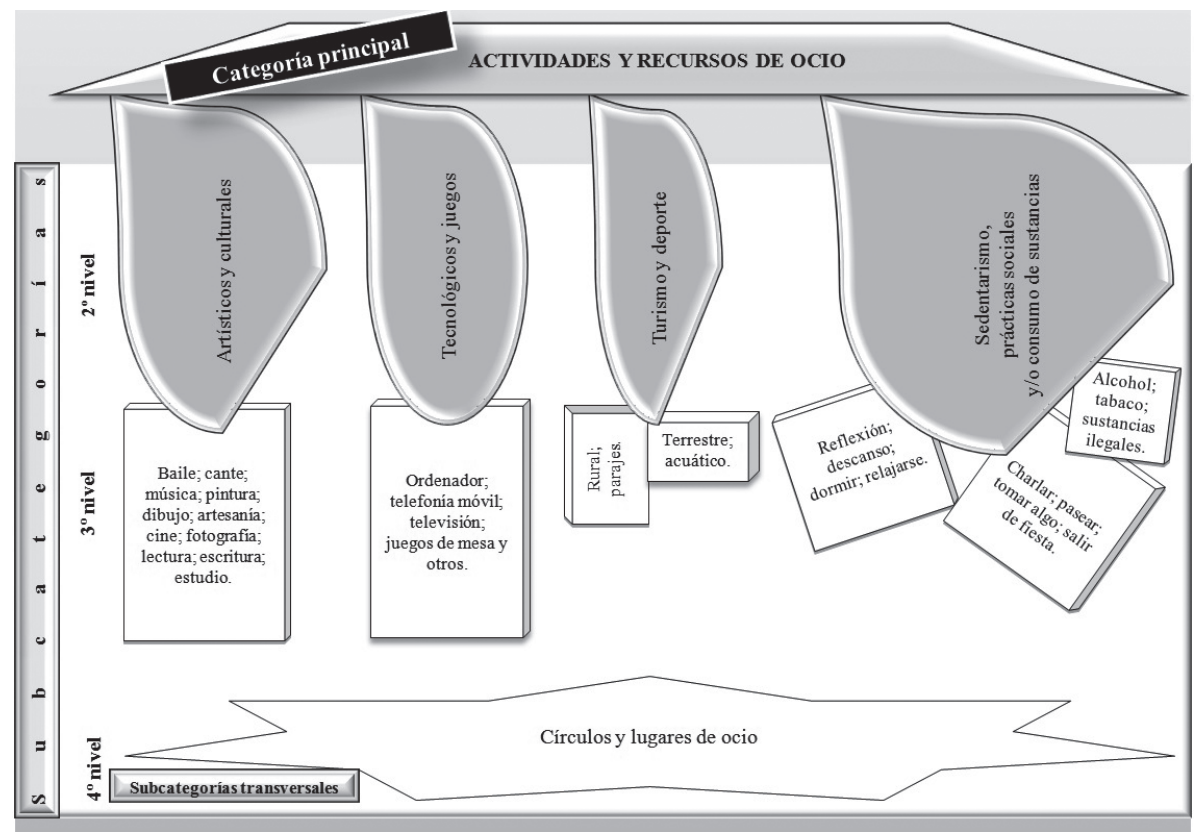

Figura 1. Panorámica sobre las actividades y recursos de ocio de los/as adolescentes.

\subsection{El ocio artístico y cultural}

Los recursos que utilizan los/as adolescentes y las actividades de ocio desarrolladas de tipo artístico y cultural evidencian cierta variedad. Un pequeño grupo se decanta por aquellas en las que emplean las manualidades de forma creativa. Como muestra hacen alusión a las prácticas que realizan de pintura y dibujo. Por otra parte, algunas aficiones de carácter artístico y artesanal, que abarcan en el tiempo libre, revelan una escasa afluencia. Con todo, a modo de ejemplo se puede mencionar la fotografía. Una adolescente expresa lo siguiente: "Cuando me apetece relajarme me dedico a pintar y dibujar o también a bailar con mis amigas. Estas actividades de ocio son mis favoritas" (Relato53: mujer, 17 años, $4^{\circ}$ curso).

Las prácticas artísticas, de carácter expresivo y musical, son desarro- 
Iladas con bastante asiduidad por algunos/as participantes. De hecho, de los resultados obtenidos se desprende que bailar y cantar les resulta muy agradable. La música constituye un elemento crucial en el ocio del colectivo analizado, ya que más de la mitad indica que incluye su escucha dentro del tiempo de libre disposición. En distintas narraciones transmiten su gusto por esta afición y detallan minuciosamente sus estilos musicales preferidos, destacando el: pop, heavy, gospel, hardcore, house, tecno o baladas. Distintos/as adolescentes, incluso, describen a sus artistas favoritos y se conciben como seguidores de los mismos. En las narraciones manifiestan que le entusiasma escuchar música en directo y que asisten a los conciertos más próximos que se celebran en la zona en la que residen (figura 2).

Los/as adolescentes experimentan una gran satisfacción tocando instrumentos musicales. En diferentes relatos concretan el instrumento por el cual sienten afición. Entre éstos figura alguno de viento (saxofón, clarinete y gaita gallega) y de cuerda (la guitarra y el piano). Otros/as participantes dejan constancia de que acuden a clase en el conservatorio de música profesional y/o se encuentran dentro de grupos o de bandas musicales locales. Como iniciativa poco común una chica revela que desarrolla composiciones musicales y las integra, con total normalidad, como parte de su ocio. En la línea de lo referido se ofrece un extracto de lo expuesto por esta adolescente:

En mi tiempo libre me gusta realizar cualquier actividad relacionada con la música, ya que me relaja y al mismo tiempo me marco un reto. Muchas tardes, que tengo libres de Instituto, las utilizo para ir al conservatorio y esto supone un gran aliciente para mí. Toco el piano también cuando me quedo en casa o enchufo mi guitarra y compongo cualquier cosa que se me venga a la cabeza. Otra de mis grandes aficiones es ir a los conciertos que se celebran cerca de mi casa para disfrutar del impresionante directo (Relato63: mujer, 15 años, $4^{\circ}$ curso). 


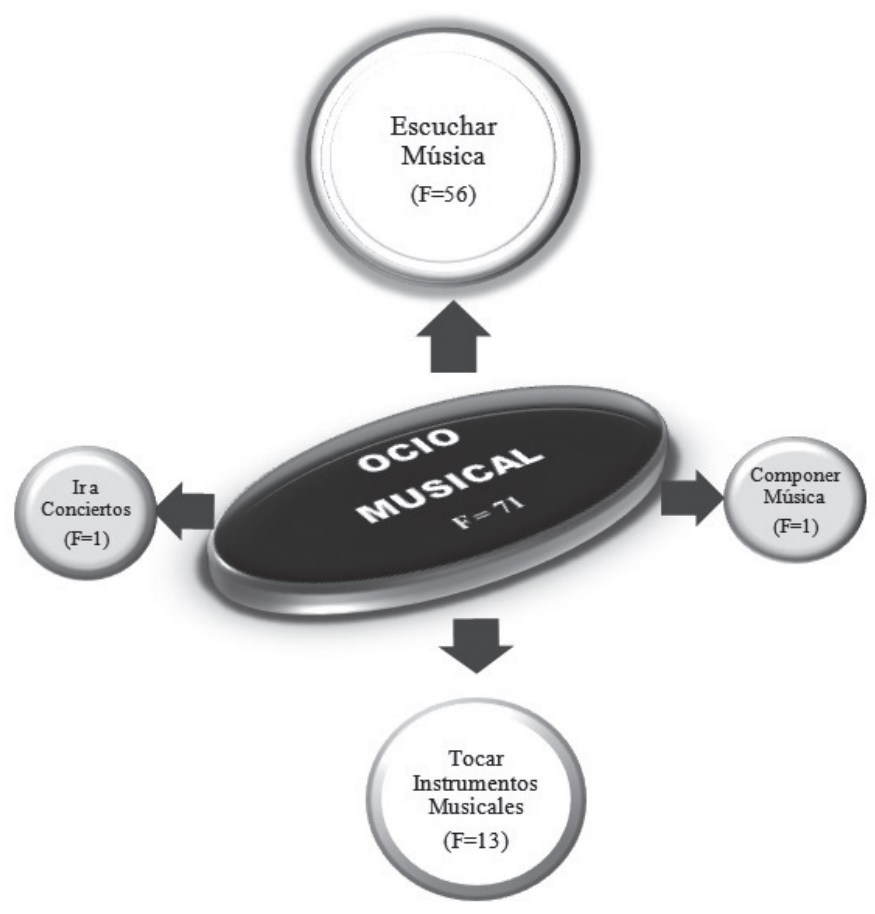

Figura 2. Preferencias en el ocio musical.

En lo relativo a otras prácticas culturales, con las que experimentan placer, señalan la lectura. Un pequeño grupo manifiesta atracción por los libros de ficción y de aventura. A este tipo de lectura dedican algún tiempo, generalmente, una vez concluidas las tareas escolares y/o en periodos vacacionales. A modo de ejemplo, cabe indicar que una chica plasma en su narración que le resulta interesante y que integra como parte de su ocio la lectura de periódicos deportivos de tirada nacional, como el Marca y el As. Cabe señalar que muy pocos utilizan las actividades de escritura como un elemento de ocio, excepcionalmente una adolescente afirma que lleva años redactando un libro y que para su creación utiliza espacios abiertos, parques o jardines públicos como medios de inspiración.

Sorprendentemente el tiempo dedicado al estudio es percibido, por una pequeña parte del colectivo, como una práctica productora de ocio. Por ejemplo, uno de los participantes indica en su relato que estudiar en alguna asignatura le resultar fascinante. En este sentido también otra adolescente apunta que disfruta buscando información relacionada con 
sus estudios. Por otra parte, la pasión por el cine la reflejan únicamente dos participantes. Además, en una línea similar, cinco chicos plasman su fascinación por el visionado de películas. Con todo, globalmente se descubre que los/as adolescentes no presentan una afición sustancial por los filmes.

\subsection{El ocio tecnológico y/o con juegos}

Las Tecnologías de la Información y Comunicación (TIC) representan un gran atractivo para los/as adolescentes y, en buena medida, las integran con las experiencias cotidianas de ocio. De hecho ponen de manifiesto la inclusión en su ocio de recursos digitales como el ordenador, el móvil y algunos programas de televisión. Un pequeño grupo, que utiliza el ordenador para el ocio, no llega a precisar las actividades que desarrolla con el mismo; mientras que en otros casos especifican el uso del chat y la navegación indiscriminada, sin un objetivo claro, a través de Internet. $\mathrm{Al}$ respecto una adolescente manifiesta:

Por la semana, después del Instituto, suelo reunirme con mis amigos y hablamos de nuestras cosas. Tenemos muchas actividades de ocio en común. Vamos al 'cyber' a jugar con videojuegos, chateamos un poco, navegamos por mil sitios en la red y muchas cosas más (Relato2: varón, 15 años, $3^{\circ}$ curso).

Algunos/as participantes utilizan el ordenador, tanto de forma individual como grupal, en este último caso reuniéndose presencialmente para jugar a videojuegos con sus coetáneos. De modo excepcional indican que le gusta jugar con otras personas en línea, aunque le resulta mucho más entretenido e interesante compartir la experiencia con sus amistades. Cabe indicar que un grupo de adolescentes plasma su pasión por las videoconsolas. Además, de forma minoritaria, se refieren a la utilización del ordenador como recurso de ocio para descargar juegos y música.

Por el visionado de la televisión los/as adolescentes muestran un uso asiduo. Es de resaltar que el recurso de la televisión es el que más utilizan, entre los de tipo tecnológico, sin discriminar el tipo de programación que les absorbe. Una adolescente comenta lo siguiente: "En mi tiempo de ocio veo normalmente cualquier cosa en la televisión, sobre todo por las noches. Además hablo mucho con 
mis amigos por el móvil y utilizo el messenger un montón" (Relato21: mujer, 14 años, $3^{\circ}$ curso).

Sobre la comunicación con telefonía móvil, una pequeña parte de los/as participantes plasma en sus relatos que le produce experiencias de ocio muy gratificantes. Asimismo, valoran sus posibilidades para mantenerse en contacto directo y continuado con sus iguales o conocidos, independientemente de la distancia física que les separe. La conversación oral establecida con las amistades, la de mensajería y las señales con "toques" (Ilamadas de atención) representan las principales aplicaciones que realizan de este dispositivo. Por otro lado, las actividades con las redes sociales les resultan relativamente atractivas, predominando el uso de la plataforma de Tuenti y Facebook.

En cuanto a los juegos de mesa, son pocos los/as participantes que sienten preferencia por los mismos. Con todo cabe referir la predilección que reflejan por el póquer, mientras solamente uno de los adolescentes se decanta por el parchís. Otros juegos que detallan, más propios de locales públicos, son el del futbolín, billar, dardos y bolos. Asimismo, una chica revela que le apasiona componer puzles en privado y durante el fin de semana. Cabe indicar que los clásicos juegos de mesa los están sustituyendo por sus homólogos en soporte digital.

\subsection{El ocio a partir de actividades turísticas y/o deportivas}

Las actividades turísticas no suponen para los/as participantes una experiencia de ocio relevante. Sin embargo, algunos manifiestan su atracción por hacer turismo y lo engloban entre sus aficiones. Es de resaltar que estos/as adolescentes son conscientes de que la economía es un factor determinante para estas iniciativas y argumentan que no se pueden permitir con frecuencia este tipo de ocio. En las preferencias de viaje que señalan de modo excepcional (tres participantes) presentan predilección por acudir a lugares apartados y principalmente por los que le resultan menos conocidos y le posibilitan cierto sosiego. Asimismo, de forma infrecuente apuntan que las visitas a los pueblos (apartados del entorno urbano), durante algún fin de semana, representan para ellos/as un gran atractivo y le posibilitan relajarse y desconectar de la estresante cotidianidad. 


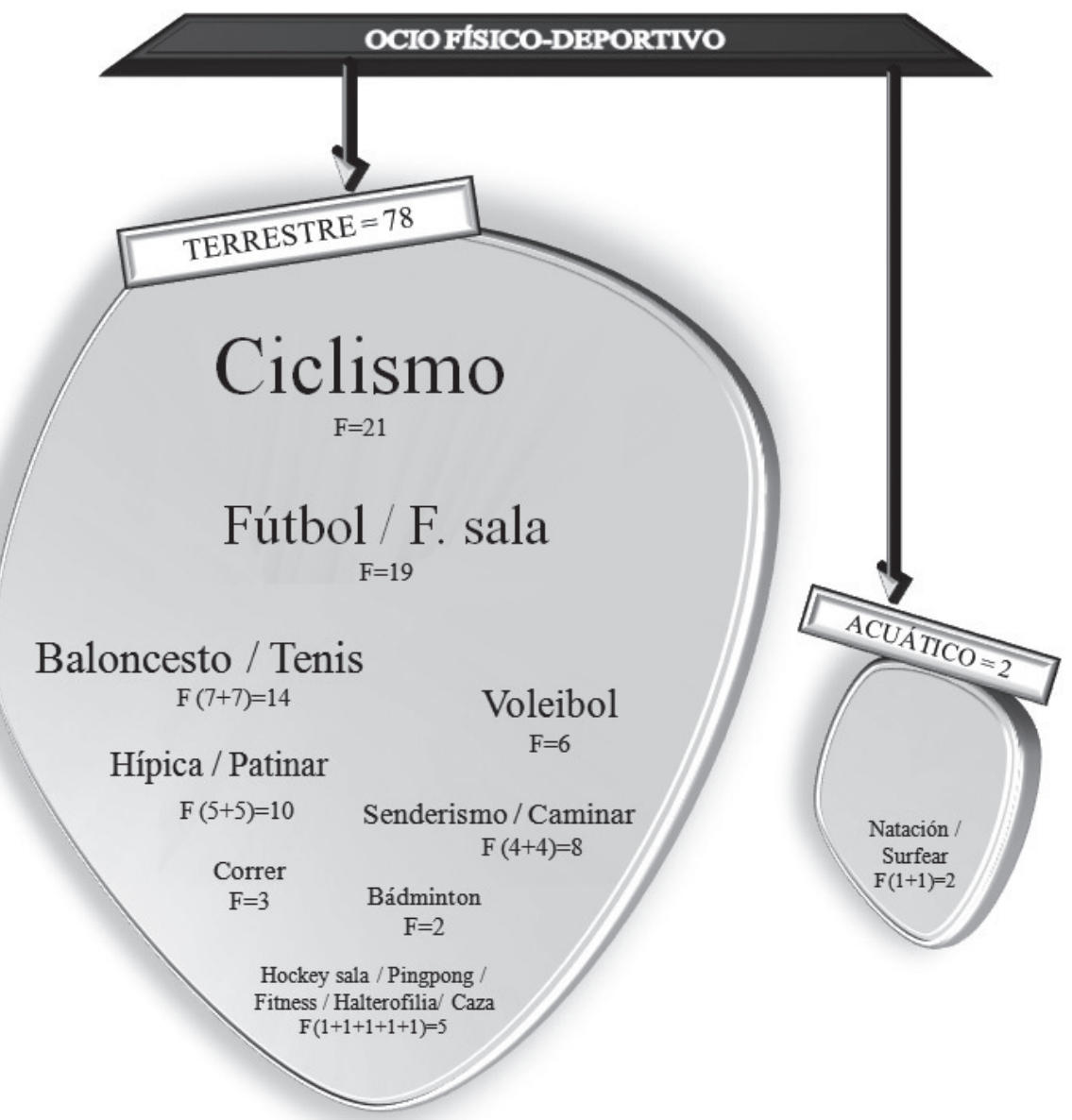

Figura 3. Tendencia en las actividades de ocio físico-deportivo.

Algunos/as participantes plasman que abarcan un abanico amplio de prácticas físico-deportivas en su tiempo libre (figura 3). De hecho, en los deportes que realizan Ilama la atención su diversidad, aunque existe la tendencia por disfrutar de los terrestres, como el ciclismo y el fútbol o el fútbol sala. También el colectivo presenta cierta preferencia por jugar al baloncesto, tenis y voleibol; de forma excepcional disfrutan del patinaje y de la hípica. Cabe indicar que una minoría se decanta por el bádminton, el ping-pong o el hockey. De modo ilustrativo se presenta el siguiente fragmento: 
Me encanta utilizar la bicicleta por el monte o con los amigos, jugar al baloncesto, al fútbol, a hockey sobre tierra, cemento y en vacaciones surfear. También me gusta ir al monte a caminar e ir al gimnasio (Relato6: varón, 14 años, $3^{\circ}$ curso).

Entre los deportes relacionados con el medio acuático, sorprende que tan sólo un adolescente revele su afición por la natación, cuando se trata de una práctica que no requiere condiciones excepcionales aunque exige una infraestructura pública o privada costosa. Con todo, generalmente a nivel público resulta económicamente asequible y su práctica es muy adecuada para el desarrollo físico de esta etapa.

De las narraciones analizadas se desprende la ausencia total de la realización de actividades de tipo aéreo, posiblemente por el riesgo que conllevan y el consecuente gasto económico. Por otro lado son de mencionar, aunque con una escasa afluencia, otras aficiones de carácter físico, por las que se decantan los/as participantes; quizá porque le resultan poco exigentes y muy asequibles, como hacer senderismo y caminar. El desarrollo de las prácticas físico-deportivas como ocio suelen tener lugar fuera del horario y del entorno escolar y las desarrollan, fundamentalmente, durante el fin de semana. Únicamente, un adolescente ha mencionado este tipo de actividades encuadrándolas en el centro educativo.

\subsection{El ocio sedentario, con prácticas sociales y/o consumo de sustancias}

En el estudio se descubre que para los/as adolescentes las actividades de ocio sedentario se limitan esencialmente a no hacer nada o a dormir. Por el acto de dormir manifiesta una notoria preferencia un pequeño grupo de participantes. El descanso también lo identifican algunos/as como una praxis ociosa que les genera tranquilidad y placer. Por otra parte, una adolescente apela a la práctica de la reflexión y otro joven a la de relajación. En este sentido, suelen aprovechar algún momento libre, por la noche o durante el fin de semana, para realizar actividades estáticas principalmente en espacios íntimos, como el salón familiar o el dormitorio. Un participante comenta: "Me gusta tumbarme en el sofá y ver la televisión, sin hacer nada más, y también dormir muchas horas sin que me despierte nadie" (Relato73: varón, 15 años, 4 curso).

El periodo de tiempo escogido por los/as adolescentes para realizar las actividades de ocio, con sus iguales, es primordialmente el del fin de 
semana. Las prácticas de ocio social que establecen en su tiempo libre con sus coetáneos son diversas. Algunos/as presentan gran interés por charlar; y lo hacen esencialmente sobre las vivencias personales propias de esta etapa o aquellos sucesos familiares, escolares o del entorno próximo relevantes y que le ocurren a lo largo de la semana. Otros/as pasean o acuden a alguna cafetería (en la que se le admite la entrada, ya que por lo general está prohibida a menores sin compañía de personas adultas), para tomarse algún refresco en compañía de sus iguales, interesándose también por hablar sobre alguna vivencia puntual.

Las fiestas populares las conciben como prácticas de ocio y las disfrutan con sus amistades en los espacios dedicados a las mismas, principalmente en lugares al aire libre. Además se detecta, en estrecha relación con algunas actividades sociales de tipo festivo, que algunos/as adolescentes se inician en el consumo de bebidas alcohólicas o sustancias prohibidas. Con todo el tabaco, las bebidas y los estupefacientes son escogidos por muy pocos/as participantes como prácticas asiduas de ocio. El fenómeno del "botellón" (en torno a la reunión de un grupo de personas, en espacios abiertos para la ingesta de alcohol) se evidencia en este estudio de forma excepcional, tan sólo por dos adolescentes, y durante el fin de semana. En este sentido, véase el siguiente fragmento: "El fin de semana, si consigo convencer a mis padres para que me dejen salir hasta tarde me voy con los amigos y hacemos el botellón, aunque eso es muy de vez en cuando" (Relato2: mujer, 15 años, $4^{\circ}$ curso).

Cabe indicar que los participantes declaran el desarrollo de diferentes prácticas de ocio en solitario y señalan otras que realizan con sus iguales. Sobre las personas con las que prefieren compartirlo, la tendencia dominante apunta a los coetáneos y, de forma determinante, con los que le une un cierto grado de amistad. La familia ocupa un tercer plano y un pequeño grupo refleja que comparte distintas actividades de ocio con ésta. Cabe resaltar que en algunos casos compaginan los miembros de la familia con sus iguales $y / 0$ amistades.

\section{Discusión y conclusiones}

Este estudio revela que las prácticas de ocio desarrolladas por los/as adolescentes y los recursos que utilizan no presentan diferencias substanciales atendiendo al género de los participantes, la edad y curso aca- 
démico en el que se encuentran. Sus preferencias irradian una aceptable diversificación y salubridad, siendo de gran utilidad su conocimiento para un enfoque educativo del ocio. De alguna forma el contexto educativo debe ser capaz de adaptarse a nuevos tiempos tratando de ofrecer nuevas prácticas, momentos, espacios curriculares (Acosta, 2009) y extracurriculares.

Cabe señalar que realizan actividades artísticas y culturales (como pintar o ir al cine) e incluyen, entre sus aficiones, de forma inusual la lectura. El bajo número de lectores, en esta etapa es un hecho, independientemente del estrato social en que se sitúen los/as adolescentes. Asimismo es preocupante por la repercusión negativa que desencadena en el ámbito curricular.

En el trabajo abordado se descubre que la utilización de recursos y actividades de tipo musical, como forma de ocio, marcan tendencia entre el colectivo. De hecho, manifiesta una aguda predilección por escuchar música y tocar instrumentos musicales de distinta naturaleza ya sea individualmente, en bandas y/o en conservatorios de música. Esto refuerza lo desvelado en otro estudio que concluye que son oyentes habituales de música (Muñoz y Olmos, 2010).

Los/as adolescentes tiene predilección por el desarrollo de actividades de ocio con las TIC, en particular chateando con los colegas y a través de la navegación por la red. El uso que realizan de las redes sociales no resulta preocupante. La preferencia que muestran por la utilización de los dispositivos digitales podría encontrarse motivada por las facilidades de manejo técnico que poseen. Además siguiendo a Torres, Robles y Molina (2011) estos resultados podrían estar influenciados por las posibilidades que tienen de acceso, ya que las personas que cursan Educación Secundaria (como es el caso) y estudios superiores, incorporan con mayor facilidad el uso individual de Internet en sus actividades académicas y cotidianas.

Se detecta una inclusión acentuada de los videojuegos en el ocio y perviven parcamente las prácticas con los juegos de mesa tradicionales. Lógicamente la afición por los primeros está influenciada por el imperioso despliegue tecnológico (Bringué, Sádaba y Sanjurjo, 2013). Lamentablemente, en ocasiones, pueden relacionarse o derivar en conductas, pensamientos y actitudes agresivas (Etxeberría, 2011). Con todo, en este estudio, se descubre que las actividades de tipo digital promueven la socialización y se encuentran vinculadas con comportamientos de inhi- 
bición y cierto aislamiento. De modo que los/as investigadores/as, educadores/as, animadores/as socioculturales han de alertar a las familias sobre los peligros de los videojuegos, en particular de los que presentan tipología violenta. Al margen de la parte negativa de los videojuegos, la escuela del siglo XXI debe estar preparada para transformar los materiales didácticos y adecuarlos a las nuevas experiencias y formas de expresarse de la sociedad, mediante estrategias de gamificación (Area y González, 2015).

El teléfono móvil es utilizado principalmente como entretenimiento: conversando entre iguales, enviándose mensajes y realizando llamadas de atención persistentes (esencialmente a los coetáneos y progenitores). Una década antes, en otra investigación (Del Pino, Duaso y Martínez, 2001) se afirmó que las nuevas tecnologías son recursos fundamentales dentro del ocio de los/as adolescentes españoles, en particular el móvil e Internet. Con el estudio aquí presentado se constata que se refuerza la tendencia de uso con este dispositivo.

Con el visionado de la televisión se pone de relieve la utilización, como actividad de ocio, de los mass media, aunque la lectura de prensa se revela como subsidiaria, al igual que la de los libros. Asimismo, en el contexto finlandés, una investigación de Pääkkönen (2005) demuestra que la televisión, junto con el ordenador, configura uno de los patrones más populares de las prácticas de ocio de los/as adolescentes. Ello, pone de manifiesto la universalidad de un patrón inactivo (más allá del contexto en el que se situó el presente estudio) a partir de la incidencia que genera, en ciertos grupos, el ocio pasivo frente al activo (como la lectura), que exige movilizar el funcionamiento e interacción de diferentes competencias cognitivas.

El turismo no ocupa un lugar importante en el escalafón de actividades de ocio en la etapa de la adolescencia. Un factor que se vislumbra como disuasorio de esta tipología de praxis es el económico. Por otro lado, el colectivo analizado siente predilección por las prácticas físicodeportivas terrestres. De hecho un estudio similar apunta que se declaran físicamente activos (Valdemoros, Sanz y Ponce, 2012), a pesar de que muchos abandonan estas experiencias antes de entrar en la etapa de la juventud, en torno a los 16 años. Además, se revela que este tipo de actividades se concentran durante el fin de semana y se desligan del entorno escolar.

Se refleja una escasa tendencia de aficiones encuadradas en un "ocio 
negativo" (nocivo o consumista) teniendo preponderancia el positivo o saludable a través del desarrollo de prácticas salpicadas de componentes educativos, creativos, lúdicos o festivos. Es de tener en consideración, principalmente por las familias y los/as profesionales de la educación y de la psicología, que el ocio practicado repercute en la calidad de vida de los/as adolescentes.

De forma extensiva las vivencias de ocio de los/as adolescentes se plasman, con mayor intensidad, a través de prácticas individuales y/o en compañía de sus iguales o amistades, siendo reducidas las desarrolladas con la familia. Para Giró (2011), ésta es una forma de liberarse de los elementos de protección y apego que, en el momento de construir la identidad, suponen un obstáculo para sus demandas de independencia. Con todo, la implicación de la familia en las actividades de ocio durante esta etapa de desarrollo es importante. Especialmente, en las actividades más propias del ocio social, se denota predilección por reunirse entre los coetáneos en lugares cerrados: domicilio familiar, bares y centros comerciales. Asociando con estos últimos el consumo de tabaco, alcohol $y / o$ estupefacientes sin que marquen una tendencia predominante en el grupo analizado. Sin embargo, desde la faceta preventiva (Vega, Aramendi y Garín, 2012) es oportuno que las familias y los/as educadores/ as permanezcan alerta ante posibles excesos, al tratarse de un potencial componente de modelo de ocio nocivo. Cabe resaltar que, en este sentido, los adultos son los principales responsables para formarles y orientarles (Camarotti, Kornblit y Di Leo, 2013).

\section{Implicaciones prácticas y limitaciones del estudio}

Los resultados y conclusiones presentadas pueden resultan de utilidad para el profesorado, la educación social y familiar con el objeto de planificar e implementar actividades de ocio, promocionando prácticas y recursos idóneos. Asimismo, podrán suscitar la reflexión, servir de punto de partida para mejorar su articulación y el desarrollo de conductas preventivas a través de la reorientación de las experiencias menos saludables o de su sustitución por otras alternativas.

La tipificación aportada sobre actividades y recursos de ocio facilitará el trabajo de los profesionales que, desde un mejor conocimiento de la realidad, podrán diseñar programas de educación para el ocio e impulsar 
intervenciones más apropiadas. Hay que tener en cuenta que en la etapa de la adolescencia educadores/as y progenitores deben jugar un papel mediador y estimular a este colectivo con opciones diversificadas y enriquecedoras. Asimismo, cabe sugerir que uno de los contextos clave para dinamizar y reforzar las actividades de ocio es el centro educativo, tanto a través de la implementación de prácticas lúdicas y creativas dentro del marco curricular (particularmente desde la materia de Educación física, las del área artística, CC. de la Naturaleza, literatura, etc.) como, en especial, a partir del extracurricular. Lo que, a su vez, contribuirá a limar la posible brecha que pueda existir en el alumnado más desfavorecido y, al mismo tiempo, reforzar la interacción o el clima de grupo y su inclusión, así como el desarrollo de la motivación y autonomía. En este sentido, es importante una oferta continuada y variada de propuestas extraescolares, junto a la disponibilidad de infraestructuras o instalaciones que resulten atractivas para el colectivo.

La transferibilidad de los resultados de esta investigación a otros contextos es susceptible de aplicación bajo una valoración reflexiva y analítica (Sthenhouse, 1987). Con todo, éste no es el fin principal de la investigación cualitativa, sino la comprensión de una determinada realidad desde su propia singularidad con el objeto de facilitar su conocimiento (González Cubillán, 2009). Si bien, a partir de este trabajo se puede ampliar su universalidad a través de un estudio longitudinal.

\section{Referencias bibliográficas}

Acosta, F. (2009). Re-configuración de la escuela secundaria frente al ingreso masivo de jóvenes de sectores populares. Revista de Pedagogía, 30(87), 217-246.

Area, M. y González, C. (2015). De la enseñanza con libros de texto al aprendizaje en espacios online gamificados. Educatio Siglo XXI, 33(3), 15-38.

Ballesta, F. J. y Cerezo, M. C. (2011). Familia y escuela ante la incorporación de las tecnologías de la información y la comunicación. Educación XX1, 14(2), 133-156.

Bringué, X., Sádaba, C. y Sanjurjo, E. (2013). Menores y ocio digital en el siglo XXI. Análisis exploratorio de perfiles de usuarios de videojuegos en España. Bordón, 65(1), 147-164.

Camarotti, A. C., Kornblit, A. L. y Di Leo, P. F. (2013). Prevención del consumo problemático de drogas en la escuela: estrategia de formación docente en Argentina utilizando TIC. Interface, 17(46), 695-703.

Castrechini, Á., Pestana, J. V. y Codina, N. (2015). El ocio juvenil en las imágenes de la 
prensa: entre la estereotipia y la ambigüedad. Pedagogía Social. Revista Interuniversitaria, 25, 91-118.

Del Pino, J. A., Duaso, A. y Martínez, R. (2001). Prácticas juveniles de ocio. Prácticas y actitudes. En J. A., del Pino, A., Duaso y R. Martínez, Opiniones y actitudes. Prácticas de ocio, cambio cultural y nuevas tecnologías en la juventud española de fin de siglo (pp. 61-84). Madrid: Centro de Investigaciones Sociológicas.

Douglas, J., Hiley, E., Palen, L. A., Darling, N., Cumsille, P. y Marta, E. (2005). Exploring adolescent self-defining leisure activities and identity experiences across three countries. International Journal of Behavioral Development, 29(5), 361-370.

Eimea, R. M., Caseya, M.M., Harveya, J.T., Sawyera, N.A., Symonsb, C. M. y Payneb, N. R. (2015). Socioecological factors potentially associated with participation in physical activity and sport: A longitudinal study of adolescent girls. Journal of Science and Medicine in Sport, 18(6), 684-690.

Etxeberría, F. (2011). Videojuegos violentos y agresividad. Pedagogía Social. Revista Interuniversitaria, 18, 31-39.

Ferreira, V, Andrade, C., Fontes, A. M., Araújo, M. C. y Anjos, S. (2015). Modos de cuidar e educar a partir do Programa de Educação pelo Trabalho para a Saúde. Interface, 19(supl.1), 857-868.

Flores, M. A. (2003). Investigar (com) os professores: reflexões sobre uma pesquisa longitudinal. Perspectiva, 21(2), 391-412.

Gabelas, J. A., Marta-Lazo, C. y González, P. (2015). El factor relacional en la convergencia mediática: una propuesta emergente. Anàlisi: Quaderns de Comunicació i Cultura, 53, 20-34.

Giró, J. (2011). Las amistades y el ocio de los adolescentes, hijos de la inmigración. Papers: Revista de Sociología, 96(1), 77-95.

Glorieux, I., Stevens, F. y Vandeweyer, J. (2005). Time use and well-being of Belgian adolescents: Research findings and time use evidence. Loisir et Societe, 28(2), 481-510.

González Cubillán, L. (2009). Estudio de casos bajo el enfoque transdisciplinar. Multiciencias, 9(3), 303-312.

Hernández, R., Fernández, C. y Baptista, P. (2006). Metodología de la investigación. Ciudad de México: McGraw-Hill.

Liu, Y. C. y Hsu, Y. C. (2013). Predicting adolescent deviant behaviors through data mining techniques. Educational Technology \& Society, 16(1), 295-308.

Mannell, R. C., Kaczynski, A. T. y Aronson, R. M. (2005). Adolescent participation and flow experience in physically active leisure and electronic media activities: Testing the displacement hypothesis. Loisir et Societe, 28(2), 653-675.

Mcmillan, J. H. y Schumacher, S. (2005). Investigación educativa una introducción conceptual. Madrid: Pearson.

Menchén, F. (2008). Crea tu propia vida en tu tiempo de ocio. Encuentros Multidisciplinares, 28, 1-12.

Muñoz, J. M. y Olmos, S. (2010). Adolescencia, tiempo libre y educación. Un estudio con alumnos de la ESO. Educación XX1, 13(2), 139-162.

Noriega, M. J. et al. ( 2015). Hábitos sedentarios en adolescentes escolarizados de Cantabria. RETOS. Nuevas Tendencias en Educación Física, Deporte y Recreación, 27, 3-7. 
Ortega, C., Lazcano, I. y Rocha, M. M. (2015). Espacios de ocio para jóvenes, de la monitorización a la autogestión. Pedagogía Social. Revista Interuniversitaria, 25, 69-89.

Pääkkönen, H. (2005). What do school children in Finland do with their time? Loisir et Societe, 28(2), 425-442.

Ricoeur, P. (1995). Tiempo y narración I. Configuración del tiempo en el relato histórico. Madrid: Siglo XXI.

San Martín, J., López, A. E. y Esteve, R. (1999). Dimensionalización del constructo de ocio en universitarios. Psicothema, 11(1), 113-124.

Sanz, E. y Romero, P. (2009). Creatividad y consumo en el ocio de niños y adolescentes: un estudio a través de sus dibujos. Educación y Futuro: Revista de Investigación Aplicada y Experiencias Educativas, 21, 111-128.

Stake, R. (2010). Qualitative research: Studying how things work. Nueva York: The Guilford Press.

Sthenhouse, L. (1987). La investigación como base de la enseñanza. Madrid: Morata.

Torres, C., Robles, J. M. y Molina, O. (2011). ¿Por qué usamos las tecnologías de la información y las comunicaciones? Un estudio sobre las bases sociales de la utilidad individual de Internet. Revista Internacional de Sociología, 69(2), 371-392.

Valdemoros, M. A., Sanz, E. y Ponce, A. (2012). Educación informal y ocio juvenil. El influjo de los amigos en el abandono de la práctica físico-deportiva. Pedagogía Social, 20, 203-221.

Vega, A., Aramendi, P. y Garín, S. (2012). Adolescentes y jóvenes: desde las conductas de riesgo a la inclusión social. Zerbitzuan, 52, 167-178.

\section{Nota y agradecimientos}

La presente aportación se encuadrada en una tesis doctoral sobre las prácticas y los recursos de ocio de los/as adolescentes gallegos. Aprovechamos para manifestar nuestro profundo agradecimiento a las personas participantes. 
